\title{
Managing spine lesions with spinal cord involvement surgically or conservatively
}

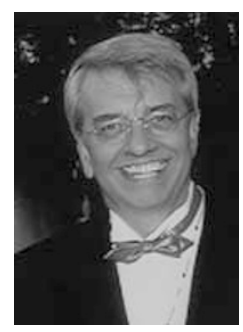

JJ Wyndaele, Editor

Antwerp University Hospital, Antwerp, Belgium

E-mail: spinalcord@uza.be

Dear Spinal Cord reader,

This September issue contains one review and two original contributions on the surgical management of spine lesions with spinal cord/cauda equina involvement because of trauma and metastatic disease.

Starting from a small case series, Dussa and Soni wrote a literature review describing how the management of transverse sacral fractures influenced bladder/bowel outcome. They tried to find statistical evidence of benefit for either a surgical or conservative management. They also listed what can be expected as conditions for poor prognosis.

Abel et al. investigated whether immediate posterior decompression and stabilization for metastatic cord compression can be recommended in patients who are able to have such surgery. Using accepted standards of documentation for spinal cord injury, a clear perspective of the results that can be expected is provided. By comparing their results with the current literature they found no evidence that anterior approaches are superior.

Singhal et al. studied the neurological outcome in patients with surgically managed incomplete closed traumatic cervical spinal cord injuries and found it comparable to the conservatively treated patients.

Flückiger et al. studied device-related complications of long-term intrathecal drug therapy through implanted pumps. They found complication rates of patients treated in their centre in the lowest ranges when compared with other published studies, and explain why.

Cardiac risk is presented in two papers. Finnie et al. found that current metabolic syndrome definitions and Framingham risk scoring may underestimate true coronary heart disease risk in people with spinal cord injury. $\mathrm{C}$ reactive protein is proposed as a potential factor to consider in the development of spinal cord injury-specific screening tools. From the same university, Gibson et al. showed that mean $\mathrm{C}$ reactive protein values in adults with chronic spinal cord injury are consistent with high coronary heart disease risk, especially in tetraplegia.

Three papers deal with topics related to urinary management: Hess et al. in a randomized, double-blind, placebo-controlled trial with a crossover design found cranberry extract tablets useful for the prevention of urinary tract infection in spinal cord-injured patients with neurogenic bladder; Schlager and Whittam found neurogenic clones, carried over weeks in the urine of asymptomatic children with neurogenic bladder, to be similar to known uropathogenic clones associated with disease; Karatas and coworkers investigated the effect of extra corporeal shock wave lithotripsy on the spinal cord in a rat model and found that it caused not only haemorrhage, but also damage to neuronal structures, except the nucleus. With extra corporeal shock wave lithotripsy used so commonly to treat urinary stones, this needs further study.

Sacral Nerve Stimulation as an option for the treatment of faecal incontinence in patients suffering from Cauda Equina Syndrome was studied by Gstaltner et al. An interesting case report is also included in this issue.

Spinal Cord (2008) 46, 589; doi:10.1038/sc.2008.109 